\title{
Preface
}

A sustained period of discovery over the past two decades has fostered a growing interest in the galactic center. This region of the sky is now the focus of many observational campaigns and an ever-growing theoretical investigation, the former because it is by far the closest (active) galactic nucleus, the latter because Sagittarius A*the supermassive black hole lurking there-offers us the most viable opportunity of studying the physics of strong fields.

One ought to approach the task of writing a book on this subject with some trepidation, knowing that what drives the excitement of new findings at the same time guarantees a rapid evolution in content. We have come far in understanding the behavior of Sagittarius A*, yet we all know that there is still much to be learned.

Unfortunately, the primary literature on this subject is now at such a mature level that young astronomers and physicists wishing to pursue its study and scientists in other disciplines find it daunting to bring themselves up to speed with current developments. My hope is that this book will assist them in their exploration.

With the many entry points created by investigators over the years, research on Sagittarius A* may at first appear to be a complex pattern of interwoven threads. I have tried to synthesize this extensive work into a crucible of essential ideas, while providing a coherent story overall. But for completeness, I have also compiled an extensive set of references to the original literature for the benefit of those wishing to study the various topics at greater depth.

I have had the good fortune over the years of being directly involved in galactic-center research, and in these endeavors I am very grateful to my students and collaborators for the pleasure of our joint efforts. They include Peter Tamblyn, Jack Hollywood, Laird Close, Sera Markoff, Alexei Khokhlov, Marco Fatuzzo, Robert Coker, Mike Fromerth, Siming Liu, Gabe Rockefeller, Brandon Wolfe, Chris Fryer, Susan Stolovy, Heino Falcke, Victor Kowalenko, Ray Volkas, Roland Crocker, Pasquale 
Blasi, Don MacCarthy, George Rieke, Benjamin Bromley, Randy Jokipii, Vahé Petrosian, Martin Pessah, Martin Prescher, Andrea Goldwurm, Guillaume Bélanger, Eric Agol, Max Ruffert, Fred Baganoff, Joe Haller, and Daniel Wang.

I am particularly grateful to my close friend and longtime collaborator Farhad Yusef-Zadeh, whose early radio images of the galactic center inspired my interest in this field and whose ongoing drive and groundbreaking observations continue to be a fountain of enthusiasm and new ideas. And for generously supporting my research in this area for more than a decade and a half, I gratefully acknowledge the National Science Foundation, the National Aeronautics and Space Administration, and the Alfred P. Sloan Foundation.

Finally, to Patricia, Marcus, Eliana, and Adrian and to my parents, whose guidance has been priceless, I extend my enduring love and gratitude.

Fulvio Melia

Tucson, Arizona 


\section{The Galactic Supermassive Black Hole}


